\title{
播种方式与种植密度互作对大穗型小麦品种产量和氮素利用率的调控 效应
}

郑飞娜初金鹏张秀费立伟 代兴龙 ${ }^{*}$ 贺明荣 ${ }^{*}$

山东农业大学农学院 / 作物生物学国家重点实验室 / 农业农村部作物生理生态与耕作重点实验室, 山东泰安 271018

摘 要: 为探明实现冬小麦进一步增产增效的调控途径, 于 2015-2016 年和 2016-2017 年连续两个生长季, 选用大 穗型品种泰农 18 , 设置 2 种播种方式(宽幅播种和常规条播)和 7 个种植密度 $\left(130 \times 10^{4} 、 200 \times 10^{4} 、 270 \times 10^{4} 、 340 \times 10^{4} 、\right.$ $410 \times 10^{4} 、 480 \times 10^{4}$ 和 $550 \times 10^{4}$ 株 $\mathrm{hm}^{-2}$ ), 研究了播种方式与种植密度互作对大穗型小麦品种产量和氮素利用率的调控 效应。结果表明, 与常规条播相比, 宽幅播种配合增密能够有效缓解单位面积穗数增加与单穗粒重降低、氮素吸收效 率提高与氮素内在利用效率下降之间的矛盾, 通过增加单位面积穗数和氮素吸收效率协同提高籽粒产量和氮素利用 率。宽幅播种条件下获得最高产量和氮素利用率的密度为 $410 \times 10^{4}$ 株 $\mathrm{hm}^{-2}$, 显著高于常规条播条件下的最优密度 $\left(340 \times 10^{4}\right.$ 株 $\left.\mathrm{hm}^{-2}\right)$, 且其增产增效幅度亦显著高于常规条播。综上所述, 宽幅播种配合合理密植具有进一步协同提高 大穗型小麦品种产量和氮素利用率的潜力。在本试验条件下, 宽幅播种(苗带宽 $8 \sim 10 \mathrm{~cm}$ )与 $410 \times 10^{4}$ 株 $\mathrm{hm}^{-2}$ 密度相 匹配是大穗型小麦品种泰农 18 获得更高产高效的最优组合。

关键词：宽幅播种；种植密度；互作效应；产量；氮素利用率

\section{Interactive effects of sowing pattern and planting density on grain yield and nitrogen use efficiency in large spike wheat cultivar}

\author{
ZHENG Fei-Na, CHU Jin-Peng, ZHANG Xiu, FEI Li-Wei, DAI Xing-Long*, and HE Ming-Rong* \\ College of Agronomy, Shandong Agricultural University / State Key Laboratory of Crop Biology / Key Laboratory of Crop Ecophysiology and Farm- \\ ing System, Ministry of Agriculture and Rural Affairs, Tai'an 271018, Shandong, China
}

\begin{abstract}
In order to find out the way to achieve further improvement in the grain yield (GY) and nitrogen use efficiency (NUE) of winter wheat, two sowing pattern (the wide range sowing and conventional drilling sowing) and seven planting densities $\left(130 \times 10^{4}, 200 \times 10^{4}, 270 \times 10^{4}, 340 \times 10^{4}, 410 \times 10^{4}, 480 \times 10^{4}\right.$, and $550 \times 10^{4}$ plants $\left.\mathrm{hm}^{-2}\right)$ were designed during $2015-2016$ and 2016-2017 growing seasons. Tainong 18, a winter wheat cultivar with larger spike and lower tillering capacity, was used to investigate the combined effects of sowing pattern and planting density on GY and NUE. Compared with the conventional drilling sowing, the wide range sowing with higher planting density effectively alleviated the negative effect of increasing spikes per unit area and nitrogen uptake efficiency (NUpE) on decreasing single spike weight and nitrogen utilization efficiency (NUtE), respectively. Concurrent improvement in GY and NUE was achieved by increasing the number of spikes per unit area and NUpE. The planting density resulting in the highest GY and NUE under wide range sowing conditions was $410 \times 10^{4}$ plants $\mathrm{hm}^{-2}$, which was significantly higher than that $\left(340 \times 10^{4}\right.$ plants $\left.\mathrm{hm}^{-2}\right)$ under conventional drilling sowing. Moreover, the increase percentage of GY and NUE under wide ranging sowing was also significantly higher than that under drilling sowing. In summary, it is feasible to further improve GY and NUE of large spike wheat cultivar through rational combination of wide range sowing with higher planting density. Under the condition of this experiment, the optimal combination measure for high GY and NUE was sowing width of $8-10 \mathrm{~cm}$ with plant density of $410 \times 10^{4}$ plants $\mathrm{hm}^{-2}$.
\end{abstract}

\footnotetext{
本研究由国家重点研发计划项目(2016YFD0300403)，国家自然科学基金青年基金(31801298)和山东省自然科学基金博士基金项目 (ZR2018BC034)资助。

This study was supported by the National Key Research and Development Program of China (2016YFD0300403), the National Natural Science Foundation of China (31801298), and the Natural Science Foundation of Shandong Province (ZR2018BC034).

*通信作者(Corresponding authors): 代兴龙, E-mail: adaisdny@163.com; 贺明荣, E-mail: mrhe@sdau.edu.cn, Tel: 0538-8244018

第一作者联系方式: E-mail: fnzheng123@163.com

Received (收稿日期): 2019-07-07; Accepted (接受日期): 2019-09-26; Published online (网络出版日期): 2019-10-14.

URL: http://kns.cnki.net/kcms/detail/11.1809.S.20191012.1740.006.html
} 
Keywords: wide range sowing pattern; planting density; interaction effect; grain yield; nitrogen use efficiency

种植密度是小麦生产中较易控制的栽培措施, 也是影响小麦群体结构、产量形成和氮素吸收利用 的重要因子 ${ }^{[1-2]}$ 。小麦合理密植有利于提高单位面积 穗数, 以及土壤中的根长密度, 进而提高地上部氮 素积累量和氮素吸收效率，实现小麦产量和氮利用 效率的协同提高 ${ }^{[2-3]}$ 。

小麦播种的苗带宽度显著影响植株田间分布、 单株分藍与成穗和根系生长 ${ }^{[4-5]}$ 。与常规条播(苗带宽 $2 \sim 3 \mathrm{~cm}$ )相比，宽幅播种(苗带宽 $8 \sim 10 \mathrm{~cm}$ )可通过提 高单位面积穗数和地上部氮素积累量协同提高小麦 产量和氮素利用率 ${ }^{[6-7]}$ 。

大穗型品种以其穗粒重高和产量潜力大在小麦 高产创建中被广泛应用 ${ }^{[6,8]}$ 。但由于其分藍成穗率低, 单位面积成穗数少，限制了其产量的进一步提高。

目前生产中多采用增加基本苗数以提高单位面积成 穗数的增产途径 ${ }^{[9]}$ 。然而, 在常规条播条件下, 过高 的基本苗数必然导致单株营养面积降低, 部分植株 因生长不良而遭淘汰，单位面积成穗数并不能显著 增加 ${ }^{[10]}$ 。因此, 依靠增加基本苗数进一步增加其单 位面积穗数的潜力已很小。宽幅播种、合理密植均 可增加大穗型小麦品种单位面积穗数, 进而提高产

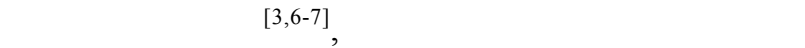

单独效应的基础上进一步提高单位面积穗数, 实现 增产增效尚末明确。

本研究设计播种方式(宽幅播种和常规条播)与 种植密度互作试验, 以期通过分析互作效应对大穗 型小麦品种产量形成和氮素吸收利用的影响, 探明 与宽幅播种相匹配的适宜种植密度和增产提效潜 力, 为充分发挥宽幅播种的增产提效作用, 实现产 量和氮素利用效率在更高水平上的协同提高提供理 论依据。

\section{1 材料与方法}

\section{1 试验点概况}

山东省泰安市岱岳区大汶口镇东武村地处温带 大陆性季风气候区, 年均日照时数 $2639 \mathrm{~h}$, 太阳辐 射总量 $119.9 \mathrm{kcal} \mathrm{cm}^{-2}$; 年均气温 $12.9^{\circ} \mathrm{C}$, 无霜期 $195 \mathrm{~d}$, 降雨量 $702 \mathrm{~mm}$, 但多数降雨集中在夏季。试 验地前茬作物为玉米, 一年两熟种植, 多年秸秆还 田。试验地土壤类型为壤土, $\mathrm{pH}$ 为 8.25 。2015-2016 年和 2016-2017 年 2 个小麦生长季播前 $1.00 \mathrm{~m}$ 土 层内无机态氮(硝态氮和铵态氮)积累量分别为 $175.43 \mathrm{~kg} \mathrm{hm}^{-2}$ 和 $186.24 \mathrm{~kg} \mathrm{hm}^{-2}$, 播前 $0 \sim 0.40 \mathrm{~m}$ 土 壤基础地力数据如表 1 所示。

表 1 2015-2016 和 2016-2017 生育季冬小麦播前 0 0.20 $\mathrm{m}$ 和 0.20 0.40m 土层基础地力

Table 1 Nutrient status of top 0-0.20 $\mathrm{m}$ and $0.20-0.40 \mathrm{~m}$ soil before seeding in 2015-2016 and 2016-2017

\begin{tabular}{cccccccc}
\hline $\begin{array}{c}\text { 年份 } \\
\text { Year }\end{array}$ & $\begin{array}{c}\text { 土层 } \\
\text { Soil layer } \\
(\mathrm{m})\end{array}$ & $\begin{array}{c}\text { 容重 } \\
\text { Bulk density } \\
\left(\mathrm{g} \mathrm{cm}^{-3}\right)\end{array}$ & $\begin{array}{c}\text { 有机质 } \\
\text { Organic matter } \\
\left(\mathrm{g} \mathrm{kg}^{-1}\right)\end{array}$ & $\begin{array}{c}\text { 全氮 } \\
\text { Total N } \\
\left(\mathrm{g} \mathrm{kg}^{-1}\right)\end{array}$ & $\begin{array}{c}\text { 碱解氮 } \\
\text { Alkali-hydrolysable N } \\
\left(\mathrm{mg} \mathrm{kg}^{-1}\right)\end{array}$ & $\begin{array}{c}\text { 速效磷 } \\
\text { Available P } \\
\left(\mathrm{mg} \mathrm{kg}^{-1}\right)\end{array}$ & $\begin{array}{c}\text { 速效钾 } \\
\text { Available K } \\
\left(\mathrm{mg} \mathrm{kg}^{-1}\right)\end{array}$ \\
\hline \multirow{2}{*}{$2015-2016$} & $0-0.20$ & 1.33 & 14.67 & 1.15 & 77.98 & 30.84 & 90.31 \\
& $0.20-0.40$ & 1.35 & 12.25 & 0.83 & 33.70 & 60.20 & 6.63 \\
$2016-2017$ & $0-0.20$ & 1.32 & 16.98 & 1.23 & 48.10 & 31.24 & 60.29 \\
& $0.20-0.40$ & 1.35 & 13.25 & 0.90 & & 60.18 \\
\hline
\end{tabular}

\section{2 试验设计}

选用大穗型品种泰农 18 为试验材料, 随机区组 设计，设宽幅播种(苗带宽 $8 \sim 10 \mathrm{~cm}$ )与常规条播(苗 带宽 2 3 cm) 2 种播种方式，每个播种方式下设 7 个 种植密度 $130 \times 10^{4} 、 200 \times 10^{4} 、 270 \times 10^{4} 、 340 \times 10^{4}$ 、 $410 \times 10^{4} 、 480 \times 10^{4}$ 和 $550 \times 10^{4}$ 株 $\mathrm{hm}^{-2}, 3$ 次重复, 共 42 个小区，小区面积为长 $15.0 \mathrm{~m} \times$ 宽 $3.0 \mathrm{~m}$ (12 行, 行距 $25 \mathrm{~cm}$ )。

播前按纯 $\mathrm{N} 、 \mathrm{P}_{2} \mathrm{O}_{5} 、 \mathrm{~K}_{2} \mathrm{O}$ 各 $120 、 90$ 和 $90 \mathrm{~kg} \mathrm{hm}^{-2}$ 施入基肥; 在拔节初期按纯 $\mathrm{N} 120 \mathrm{~kg} \mathrm{hm}^{-2}$ 追肥。所
用氮、磷、钾肥分别为尿素、过磷酸钙、氯化钾，其 氮、磷、钾含量分别为 $46 \% 、 12 \%$ 和 $60 \%$ 。其他管 理措施与高产小麦田相同。

\section{3 测定项目与方法}

1.3.1 成熟期地上部氮素积累量 成熟期随机取 50 个单茎, 人工分为茎、叶、籽粒、颖壳+穗轴等 4 部分, 烘干至恒重后称重、粉碎, 采用凯氏定氮仪测 定氮素含量, 计算地上部氮素积累量。

1.3.2 产量和产量构成因素成熟期选取长势均 匀的 $3.0 \mathrm{~m} \times 1.5 \mathrm{~m}$ 区域调查单位面积穗数, 然后人 
工收割、脱粒、风干后称重，计算产量。成熟期随 机取 50 个单茎, 调查穗粒数、千粒重, 计算单穗 粒重。

1.3.3 氮素利用率及其相关指标计算供氮量 $\left(\mathrm{kg} \mathrm{hm}^{-2}\right)=$ 施氮量 $\left(\mathrm{kg} \mathrm{hm}^{-2}\right)+$ 播前 $0 \sim 1.00 \mathrm{~m}$ 土层 土壤无机态氮积累量 $\left(\mathrm{kg} \mathrm{hm}^{-2}\right)^{[11]}$

氮素利用率 NUE $\left(\mathrm{kg} \mathrm{kg}^{-1}\right)=$ 籽粒产量 $\left(\mathrm{kg} \mathrm{hm}^{-2}\right)$ / 供氮量 $\left(\mathrm{kg} \mathrm{hm}^{-2}\right)$

氮素吸收效率 $\mathrm{NUpE}=$ 成熟期地上部氮素积累 量 $\left(\mathrm{kg} \mathrm{hm}^{-2}\right)$ / 供氮量 $\left(\mathrm{kg} \mathrm{hm}^{-2}\right) \times 100 \%$

氮素内在利用效率 NUtE $\left(\mathrm{kg} \mathrm{kg}^{-1}\right)=$ 籽粒产量 $\left(\mathrm{kg} \mathrm{hm}^{-2}\right)$ /成熟期地上部氮素积累量 $\left(\mathrm{kg} \mathrm{hm}^{-2}\right)$

\subsection{4 各因素效应计算 ${ }^{[12]}$ (1) $\mathrm{A}_{\mathrm{i}}$ (播种方式与种} 植密度的综合增产效应, $\mathrm{kg} \mathrm{hm}^{-2}$ ) = 宽幅播种下密 度产量 $\mathrm{T}_{\mathrm{i}}$-常规条播下密度产量 $\mathrm{T}_{\mathrm{i}-1}$ 。

(2) $\mathrm{B}_{\mathrm{i}}$ (播种方式与种植密度互作的增产效应, $\mathrm{kg} \mathrm{hm}^{-2}$ ) = [播种方式与种植密度的综合增产效应 -(常规条播下密度产量 $T_{i}-$ 常规条播下密度产量 $\mathrm{T}_{\mathrm{i}-1}$ ) - (宽幅播种下密度产量 $\mathrm{T}_{\mathrm{i}-1}-$ 常规条播下密度 产量 $\left.\left.\mathrm{T}_{\mathrm{i}-1}\right)\right] / 2$

(3) $C_{i}$ (播种方式与种植密度的综合增效效应, $\mathrm{kg} \mathrm{hm}^{-2}$ ) $=$ 宽幅播种下密度氮素利用率 $\mathrm{T}_{\mathrm{i}}-$ 常规条 播下密度氮素利用率 $\mathrm{T}_{\mathrm{i}-1}$ 。

(4) $D_{i}$ (播种方式与种植密度互作的增效效应, $\mathrm{kg} \mathrm{hm}^{-2}$ ) = [播种方式与种植密度的综合增效效应 (常规条播下密度氮素利用率 $\mathrm{T}_{\mathrm{i}}-$ 常规条播下密度 氮素利用率 $T_{i-1}$ ) $-\left(\right.$ 宽幅播种下密度氮素利用率 $T_{i-1}-$ 常规条播下密度氮素利用率 $\left.\left.\mathrm{T}_{\mathrm{i}-1}\right)\right] / 2$

\section{4 数据处理与统计}

采用 Microsoft Excel 2007 和 DPS 7.05 数据分析 软件整理与分析数据, 采用 LSD 判别法进行差异显 著性检验，采用 SigmaPlot 10.0 和 Microsoft Word 2007 软件作图。

\section{2 结果与分析}

2.1 各关键指标方差分析结果

方差分析表明(表 2)，播种方式、种植密度及两 者的互作效应显著影响大穗型小麦产量、产量构成 因素、氮素利用率、氮素吸收效率和氮素内在利用 效率。鉴于播种方式、种植密度的单独效应前人已 有较多研究, 本论文中将重点分析并讨论播种方式 和种植密度的互作效应。

表 2 年份 $(Y)$ 、播种方式(S)与种植密度(D)对大穗型小麦品种产量、产量构成因素、氮素利用率及其构成因素影响的方差分析

Table 2 Variance analysis of grain yield, yield components, nitrogen use efficiency and its components as affected by year (Y), sowing pattern (S), planting density (D) in large spike wheat cultivar

\begin{tabular}{lcccccc}
\hline \multicolumn{1}{c}{$\begin{array}{c}\text { 变异来源 } \\
\text { Source of variation }\end{array}$} & $\begin{array}{c}\text { 籽粒产量 } \\
\text { Grain yield }\end{array}$ & $\begin{array}{c}\text { 单位面积穗数 } \\
\text { Spikes per unit area }\end{array}$ & $\begin{array}{c}\text { 穗粒数 } \\
\text { Kernels per } \\
\text { spike }\end{array}$ & $\begin{array}{c}\text { 千粒重 } \\
1000 \text {-kernel weight }\end{array}$ & $\begin{array}{c}\text { 氮素利用率 } \\
\text { NUE }\end{array}$ & $\begin{array}{c}\text { 氮素吸收效 } \\
\text { 率 }\end{array}$ NUpE 氮素内在利用 \\
效率 NUtE
\end{tabular}

${ }^{*},{ }^{* *}$ 和 ${ }^{* *}$ 分别代表 $P<0.05 、 P<0.01$ 和 $P<0.001$ 显著水平。NUE: 氮素利用率; NUpE: 氮素吸收效率; NUtE: 氮素内在利用效率。

${ }^{*},{ }^{* *}$, and ${ }^{* * *}$ : significant at $P<0.05, P<0.01$, and $P<0.001$, respectively. NUE: nitrogen use efficiency; NUpE: nitrogen uptake efficiency; NUtE: nitrogen utilization efficiency.

2.2 播种方式与种植密度互作对大穗型小麦产 量及产量构成因素的影响

2.2.1 播种方式与种植密度互作对大穗型小麦产量 的影响 如图 1-a, b 所示, 两生长季常规条播下种 植密度为 $340 \times 10^{4}$ 株 $\mathrm{hm}^{-2}$ 时产量达到最高, 较最低的 $130 \times 10^{4}$ 株 $\mathrm{hm}^{-2}$ 密度平均增产 $1459.04 \mathrm{~kg} \mathrm{hm}^{-2}$, 增幅 $18.57 \%$ 。宽幅播种下均在 $410 \times 10^{4}$ 株 $\mathrm{hm}^{-2}$ 密度产量达
到最高, 较最低密度平均增产 $2095.04 \mathrm{~kg} \mathrm{hm}^{-2}$, 增幅 $26.70 \%$ 。宽幅播种下获得最高产量的密度以及合理 密植的增产幅度均高于常规条播。

两生长季宽幅播种与种植密度的综合增产效应 随种植密度的提高而降低。当密度由 $130 \times 10^{4}$ 株 $\mathrm{hm}^{-2}$ 增至 $410 \times 10^{4}$ 株 $\mathrm{hm}^{-2}$ 时, 综合增产效应由 1332.07 降低至 $1139.30 \mathrm{~kg} \mathrm{hm}^{-2}$ ，降幅 $14.47 \%$; 当密 
度由 $410 \times 10^{4}$ 株 $\mathrm{hm}^{-2}$ 进一步增至 $550 \times 10^{4}$ 株 $\mathrm{hm}^{-2}$ 时, 综合增产效应由 $1139.30 \mathrm{~kg} \mathrm{hm}^{-2}$ 降低至 $908.60 \mathrm{~kg} \mathrm{hm}^{-2}$, 降幅 20.25\% (图 1-c, d)。

两生长季宽幅播种与种植密度互作的增产效 应随种植密度的提高呈现先增加后降低的趋势。 当密度由 $130 \times 10^{4}$ 株 $\mathrm{hm}^{-2}$ 增加至 $410 \times 10^{4}$ 株 $\mathrm{hm}^{-2}$
时, 互作增产效应从 $55.88 \mathrm{~kg} \mathrm{hm}^{-2}$ 增至 $212.38 \mathrm{~kg}$ $\mathrm{hm}^{-2}$, 然而当密度由 $410 \times 10^{4}$ 进一步增至 $550 \times 10^{4}$ 株 $\mathrm{hm}^{-2}$ 时, 互作增产效应从 212.38 降至-11.55 $\mathrm{kg} \mathrm{hm}^{-2}$ 。互作增产效应在 $410 \times 10^{4}$ 株 $\mathrm{hm}^{-2}$ 密度时 达到最大，此时产量达到最高的 $9969.97 \mathrm{~kg}$ $\mathrm{hm}^{-2}$ (图 1-e, f)。
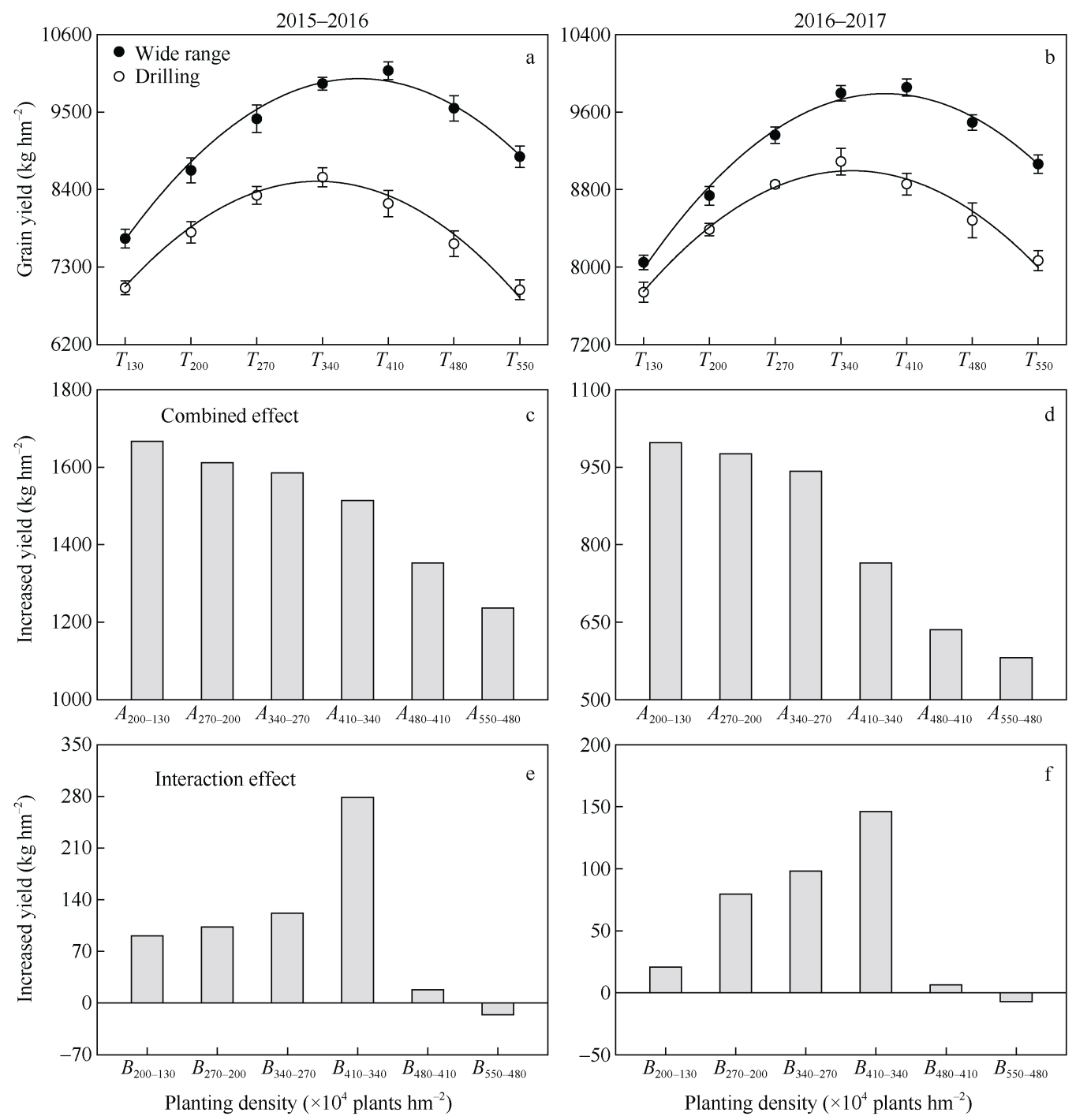

图 1 播种方式与种植密度互作对大穗型小麦品种产量的影响

Fig. 1 Effect of interaction of sowing pattern and planting density on the grain yield of large spike wheat cultivar 误差线表示 3 次重复的标准误。

The error bars represent standard error of three replicates.

\section{2 .2 播种方式与种植密度互作对大穗型小麦产量} 构成因素的影响如表 3 所示, 两生长季常规条 播下, 种植密度为 $340 \times 10^{4}$ 株 $\mathrm{hm}^{-2}$ 时单位面积穗数 最高, 较最低密度平均增穗 $194.82 \times 10^{4}$ 穗 $\mathrm{hm}^{-2}$, 增 幅 $43.23 \%$ 。宽幅播种下在 $410 \times 10^{4}$ 株 $\mathrm{hm}^{-2}$ 密度时 单位面积穗数最高, 较最低密度平均增穗 $295.15 \times$ $10^{4}$ 穗 $\mathrm{hm}^{-2}$, 增幅 $65.49 \%$ 。两播种方式下, 密度进
一步提高时穗数不再显著增加。

如表 3 所示, 随种植密度增加穗粒数与千粒重均 呈下降趋势。常规条播下 $340 \times 10^{4}$ 株 $\mathrm{hm}^{-2}$ 密度较最 低密度的穗粒数、千粒重分别降低 5.82 粒与 $2.39 \mathrm{~g}$, 降幅分别为 $12.13 \%$ 和 $13.28 \%$ 。宽幅播种下 $410 \times 10^{4}$ 株 $\mathrm{hm}^{-2}$ 密度较最低密度的穗粒数、千粒重分别降低 6.56 粒和 $3.03 \mathrm{~g}$, 降幅分别为 $13.28 \%$ 和 $7.59 \%$ 。 
表 3 播种方式与种植密度互作对大穗型小麦品种产量和氮素利用率构成因素的影响

Table 3 Effects of sowing pattern (S), planting density (D) on components of grain yield and nitrogen use efficiency in large spike wheat cultivar

\begin{tabular}{|c|c|c|c|c|c|c|c|}
\hline $\begin{array}{l}\text { 年份 } \\
\text { Year }\end{array}$ & $\begin{array}{c}\text { 播种方式 } \\
\text { Sowing } \\
\text { pattern }\end{array}$ & $\begin{array}{c}\text { 种植密度 } \\
\text { Plant density } \\
\left(\times 10^{4} \text { plants }\right. \\
\left.\mathrm{hm}^{-2}\right)\end{array}$ & $\begin{array}{c}\text { 穗数 } \\
\text { Spikes per unit } \\
\text { area }\left(\times 10^{4} \text { plants }\right. \\
\left.\mathrm{hm}^{-2}\right)\end{array}$ & $\begin{array}{c}\text { 穗粒数 } \\
\text { Kernels per spike } \\
\left(\text { No. spike }{ }^{-1}\right)\end{array}$ & $\begin{array}{c}\text { 千粒重 } \\
\text { 1000-kernel } \\
\text { weight (g) }\end{array}$ & $\begin{array}{c}\text { 氮素吸收效率 } \\
\mathrm{NUpE} \\
(\%)\end{array}$ & $\begin{array}{c}\text { 氮素利用效率 } \\
\text { NUtE } \\
\left(\mathrm{kg} \mathrm{kg}^{-1}\right)\end{array}$ \\
\hline \multirow[t]{14}{*}{ 2015-2016 } & 宽幅播种 & T130 & $451.05 \mathrm{f}$ & $49.40 \mathrm{a}$ & $39.96 \mathrm{ef}$ & $55.73 \mathrm{i}$ & $33.27 \mathrm{~b}$ \\
\hline & Wide range & T200 & $540.07 \mathrm{de}$ & $47.48 \mathrm{bc}$ & $39.61 \mathrm{fg}$ & $68.23 \mathrm{e}$ & $30.70 \mathrm{de}$ \\
\hline & & $\mathrm{T} 270$ & $622.61 \mathrm{c}$ & $45.63 \mathrm{~cd}$ & $39.12 \mathrm{~h}$ & $76.88 \mathrm{~d}$ & $29.44 \mathrm{fg}$ \\
\hline & & T340 & $696.65 \mathrm{~b}$ & $43.55 \mathrm{e}$ & $38.62 \mathrm{i}$ & $83.52 \mathrm{~b}$ & $28.54 \mathrm{gh}$ \\
\hline & & $\mathrm{T} 410$ & $748.65 \mathrm{a}$ & $41.57 \mathrm{fg}$ & $38.13 \mathrm{ij}$ & $86.18 \mathrm{a}$ & $28.27 \mathrm{~h}$ \\
\hline & & $\mathrm{T} 480$ & $778.02 \mathrm{a}$ & $38.22 \mathrm{~h}$ & $37.72 \mathrm{j}$ & $81.67 \mathrm{c}$ & $28.15 \mathrm{~h}$ \\
\hline & & T550 & $783.83 \mathrm{a}$ & $35.99 \mathrm{i}$ & $37.20 \mathrm{k}$ & $76.41 \mathrm{~d}$ & $27.90 \mathrm{~h}$ \\
\hline & 传统条播 & $\mathrm{T} 130$ & $388.67 \mathrm{~g}$ & $47.95 \mathrm{ab}$ & $42.99 \mathrm{a}$ & $46.57 \mathrm{j}$ & $36.21 \mathrm{a}$ \\
\hline & Drilling & $\mathrm{T} 200$ & $460.33 \mathrm{f}$ & $45.50 \mathrm{~d}$ & $42.68 \mathrm{a}$ & $55.98 \mathrm{hi}$ & $33.50 \mathrm{~b}$ \\
\hline & & $\mathrm{T} 270$ & $529.00 \mathrm{e}$ & $43.05 \mathrm{ef}$ & $42.17 \mathrm{~b}$ & $62.71 \mathrm{~g}$ & $31.92 \mathrm{c}$ \\
\hline & & T340 & $583.33 \mathrm{~cd}$ & $40.73 \mathrm{~g}$ & $41.58 \mathrm{c}$ & $66.90 \mathrm{e}$ & $31.04 \mathrm{~cd}$ \\
\hline & & $\mathrm{T} 410$ & $600.63 \mathrm{c}$ & $38.06 \mathrm{~h}$ & $41.06 \mathrm{~d}$ & $65.17 \mathrm{f}$ & $30.79 \mathrm{de}$ \\
\hline & & $\mathrm{T} 480$ & $608.50 \mathrm{c}$ & $35.46 \mathrm{i}$ & $40.19 \mathrm{e}$ & $61.43 \mathrm{~g}$ & $29.70 \mathrm{ef}$ \\
\hline & & T550 & $611.03 \mathrm{c}$ & $33.02 \mathrm{~g}$ & $39.36 \mathrm{gh}$ & $57.56 \mathrm{~h}$ & $28.17 \mathrm{~h}$ \\
\hline \multirow[t]{14}{*}{ 2016-2017 } & 宽幅播种 & T130 & $450.27 \mathrm{~h}$ & $45.30 \mathrm{bc}$ & $42.28 \mathrm{a}$ & $63.44 \mathrm{~h}$ & $29.76 \mathrm{c}$ \\
\hline & Wide range & $\mathrm{T} 200$ & $554.33 \mathrm{f}$ & $44.28 \mathrm{~cd}$ & $41.01 \mathrm{~b}$ & $72.36 \mathrm{e}$ & $28.32 \mathrm{ef}$ \\
\hline & & $\mathrm{T} 270$ & $646.38 \mathrm{~d}$ & $42.39 \mathrm{e}$ & $39.91 \mathrm{c}$ & $78.62 \mathrm{~d}$ & $27.70 \mathrm{fg}$ \\
\hline & & T340 & $699.82 \mathrm{c}$ & $41.11 \mathrm{f}$ & $38.92 \mathrm{~d}$ & $83.73 \mathrm{~b}$ & $27.29 \mathrm{gh}$ \\
\hline & & $\mathrm{T} 410$ & $742.96 \mathrm{ab}$ & $40.01 \mathrm{~g}$ & 38.04 ef & $85.80 \mathrm{a}$ & $27.14 \mathrm{ghi}$ \\
\hline & & $\mathrm{T} 480$ & $767.69 \mathrm{a}$ & $38.43 \mathrm{~h}$ & $37.35 \mathrm{gh}$ & $83.72 \mathrm{~b}$ & $26.96 \mathrm{hi}$ \\
\hline & & T550 & $779.03 \mathrm{a}$ & $37.33 \mathrm{i}$ & $36.84 \mathrm{hi}$ & $81.14 \mathrm{c}$ & $26.43 \mathrm{i}$ \\
\hline & 传统条播 & T130 & $413.99 \mathrm{i}$ & $46.92 \mathrm{a}$ & $41.19 \mathrm{~b}$ & $58.00 \mathrm{i}$ & $31.92 \mathrm{a}$ \\
\hline & Drilling & $\mathrm{T} 200$ & $498.27 \mathrm{~g}$ & $45.70 \mathrm{~b}$ & $39.95 \mathrm{c}$ & $64.28 \mathrm{~h}$ & $30.61 \mathrm{~b}$ \\
\hline & & $\mathrm{T} 270$ & $572.71 \mathrm{f}$ & $43.71 \mathrm{~d}$ & $38.59 \mathrm{de}$ & $68.96 \mathrm{~g}$ & $29.91 \mathrm{bc}$ \\
\hline & & T340 & $608.97 \mathrm{de}$ & $42.50 \mathrm{e}$ & $37.83 \mathrm{fg}$ & $72.91 \mathrm{e}$ & $29.42 \mathrm{~cd}$ \\
\hline & & $\mathrm{T} 410$ & $635.73 \mathrm{~d}$ & $41.25 \mathrm{f}$ & $36.63 \mathrm{i}$ & $71.66 \mathrm{f}$ & $28.96 \mathrm{e}$ \\
\hline & & $\mathrm{T} 480$ & $649.79 \mathrm{~d}$ & $39.31 \mathrm{gh}$ & $35.72 \mathrm{j}$ & $70.23 \mathrm{fg}$ & $27.78 \mathrm{fg}$ \\
\hline & & T550 & $654.84 \mathrm{~d}$ & $38.30 \mathrm{hi}$ & $35.21 \mathrm{j}$ & $69.22 \mathrm{~g}$ & $26.54 \mathrm{i}$ \\
\hline
\end{tabular}

多重比较仅在同一列同一年份间进行, 不同字母代表在 $P<0.05$ 水平上差异显著。NUpE: 氮素吸收效率; NUtE: 氮素内在利用效率。 Values followed by the same letter within a column in the same year are not significantly different at $P<0.05$ as determined by the LSD test. NUpE: nitrogen uptake efficiency; NUtE: nitrogen utilization efficiency.

\section{3 播种方式与种植密度互作对冬小麦氮素利} 用率及其构成因素的影响

\subsection{1 播种方式与种植密度互作对冬小麦氮素利用} 率的影响 如图 2-a, b 所示, 两生长季常规条播下, 种植密度为 $340 \times 10^{4}$ 株 $\mathrm{hm}^{-2}$ 时氮素利用率达到最高, 较 最低密度平均增加 $3.47 \mathrm{~kg} \mathrm{~kg}^{-1}$, 增幅 $18.57 \%$ 。宽幅播种 下均在 $410 \times 10^{4}$ 株 $\mathrm{hm}^{-2}$ 密度时氮素利用率达到最高, 较 最低密度平均增加 $4.99 \mathrm{~kg} \mathrm{~kg}^{-1}$, 增幅 $26.70 \%$ 。宽幅播种
下获得最高氮素利用率的密度以及合理密植对氮素利用 率的提升效应均高于常规条播。

播种方式与种植密度互作对氮素利用率的提升 效应随密度的提高而降低。当密度由 $130 \times 10^{4}$ 株 $\mathrm{hm}^{-2}$ 增至 $410 \times 10^{4}$ 株 $\mathrm{hm}^{-2}$ 时, 综合效应从 $3.18 \mathrm{~kg} \mathrm{~kg}^{-1}$ 降 至 $2.72 \mathrm{~kg} \mathrm{~kg}^{-1}$, 降幅 $14.39 \%$; 当密度由 $410 \times 10^{4}$ 株 $\mathrm{hm}^{-2}$ 进一步增至 $550 \times 10^{4}$ 株 $\mathrm{hm}^{-2}$ 时, 综合效应从 $2.72 \mathrm{~kg} \mathrm{~kg}^{-1}$ 降至 $2.17 \mathrm{~kg} \mathrm{~kg}^{-1}$, 降幅 20.21\% (图 2-c, d)。 

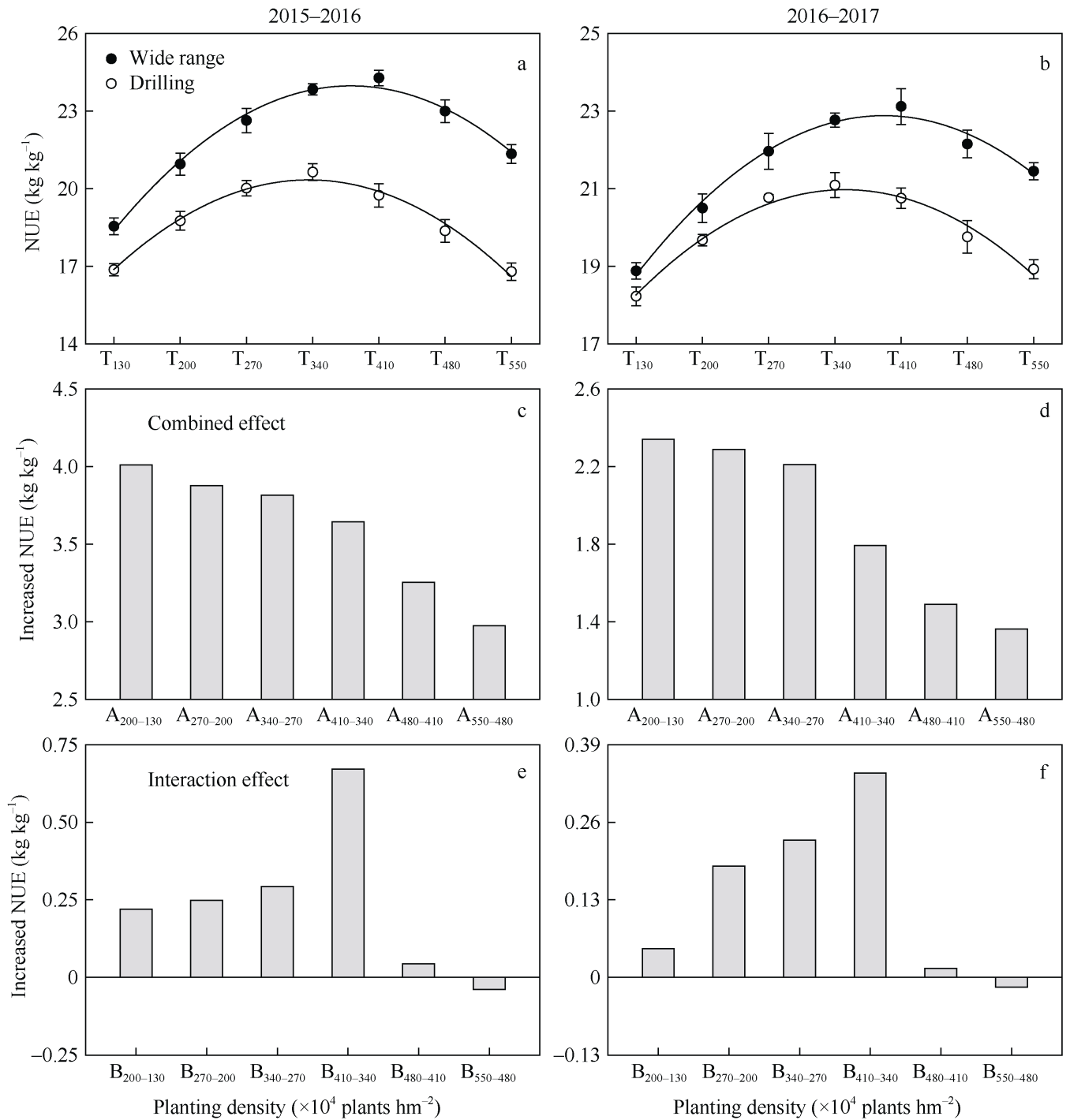

图 2 播种方式与种植密度互作对大穗型小麦品种氮素利用率(NUE)的影响

Fig. 2 Effect of interaction of sowing pattern and planting density on the nitrogen use efficiency (NUE) of large spike wheat cultivar 误差线表示 3 次重复的标准误。

The error bars represent standard error of three replicates.

播种方式与种植密度互作对氮素利用率的提升效 应随密度的提高呈先增加后降低的趋势。当密度由 $130 \times 10^{4}$ 株 $\mathrm{hm}^{-2}$ 增至 $410 \times 10^{4}$ 株 $\mathrm{hm}^{-2}$ 时, 互作的氮素 利用率提升效应从 $0.13 \mathrm{~kg} \mathrm{~kg}^{-1}$ 增至 $0.51 \mathrm{~kg} \mathrm{~kg}^{-1}$, 当密 度由 $410 \times 10^{4}$ 株 $\mathrm{hm}^{-2}$ 进一步增至 $550 \times 10^{4}$ 株 $\mathrm{hm}^{-2}$ 时, 效应从 $0.51 \mathrm{~kg} \mathrm{~kg}^{-1}$ 降至 $-0.03 \mathrm{~kg} \mathrm{~kg}^{-1}$ 。互作的氮素利 用率提升效应在 $410 \times 10^{4}$ 株 $\mathrm{hm}^{-2}$ 密度时达到最大, 此 时氮素利用率也取得最高的 $23.68 \mathrm{~kg} \mathrm{~kg}^{-1}$ (图 2-e, f)。

2.3.2 播种方式与种植密度互作对冬小麦氮素吸收 效率和内在利用效率的影响如表 3 所示, 常规 条播下密度为 $340 \times 10^{4}$ 株 $\mathrm{hm}^{-2}$ 时氮素吸收效率最高, 较最低密度平均增加 $17.62 \%$, 增幅 $30.00 \%$ 。密度进
一步提高, 氮素吸收效率呈下降趋势。宽幅播种下 $410 \times 10^{4}$ 株 $\mathrm{hm}^{-2}$ 密度氮素吸收效率最高, 较最低密 度平均增加 $26.40 \%$, 增幅 $44.94 \%$ 。密度进一步提高, 氮素吸收效率呈下降趋势。宽幅播种下氮素吸收效 率最优的密度及合理密植对氮素吸收效率的提升效 应均高于常规条播。

如表 3 所示, 宽幅播种小麦的氮素内在利用效 率低于常规条播。两播种方式下的氮素内在利用效 率均随密度增加而降低, 与最低密度相比, 常规条 播与宽幅播种下 $550 \times 10^{4}$ 株 $\mathrm{hm}^{-2}$ 密度的氮素内在利 用效率分别降低 $6.71 \mathrm{~kg} \mathrm{~kg}^{-1}$ 和 $4.35 \mathrm{~kg} \mathrm{~kg}^{-1}$, 降幅分 别为 $19.53 \%$ 和 $13.66 \%$ 。 
2.4 宽幅播种与种植密度互作对冬小麦穗数和 单穗粒重的影响

如图 3 所示, 两种播种方式下随单位面积穗数增加, 单穗粒重呈先缓慢下降后急剧下降的趋势。常规条播下, 随密度依次由 $130 \times 10^{4}$ 株 $\mathrm{hm}^{-2}$ 增至 $200 \times 10^{4} 、 270 \times 10^{4}$ 、 $340 \times 10^{4} 、 410 \times 10^{4} 、 480 \times 10^{4}$ 和 $550 \times 10^{4}$ 株 $\mathrm{hm}^{-2}$, 穗数 由 $401.33 \times 10^{4}$ 穗 $\mathrm{hm}^{-2}$ 相应升至 $479.30 \times 10^{4}$ 、 $550.85 \times 10^{4} 、 596.15 \times 10^{4} 、 618.18 \times 10^{4} 、 629.14 \times 10^{4}$ 和 $632.94 \times 10^{4}$ 穗 $\mathrm{hm}^{-2}$ 。与相邻低密度相比, 每增加 $1 \times 10^{4}$ 穗 $\mathrm{hm}^{-2}$, 单穗粒重分别下降 $1.68 、 1.80 、 1.90 、 4.97$ 、
9.83 和 $24.82 \mathrm{mg}$, 表明条播下穗数升至 $596.15 \times 10^{4}$ 穗 $\mathrm{hm}^{-2}$ 后单穗粒重急剧下降。宽幅播种下，随密度依次 由 130 株 $\mathrm{hm}^{-2}$ 增加至 $200 \times 10^{4} 、 270 \times 10^{4} 、 340 \times 10^{4}$ 、 $410 \times 10^{4} 、 480 \times 10^{4} 、 550 \times 10^{4}$ 株 $\mathrm{hm}^{-2}$, 穗数由 $450.66 \times 10^{4}$ 穗 $\mathrm{hm}^{-2}$ 相应升至 $547.20 \times 10^{4} 、 634.49 \times 10^{4} 、 698.23 \times 10^{4}$ 、 $745.81 \times 10^{4} 、 772.85 \times 10^{4}$ 和 $781.43 \times 10^{4}$ 穗 $\mathrm{hm}^{-2}$ 。与相邻 低密度相比, 每增加 $1 \times 10^{4}$ 穗 $\mathrm{hm}^{-2}$, 单穗粒重分别下 降 1.22、1.31、1.33、1.40、4.07 和 $14.33 \mathrm{mg}$ ，表明宽 播播种下穗数升至 $745.81 \times 10^{4}$ 穗 $\mathrm{hm}^{-2}$ 后单穗粒重才 快速下降。
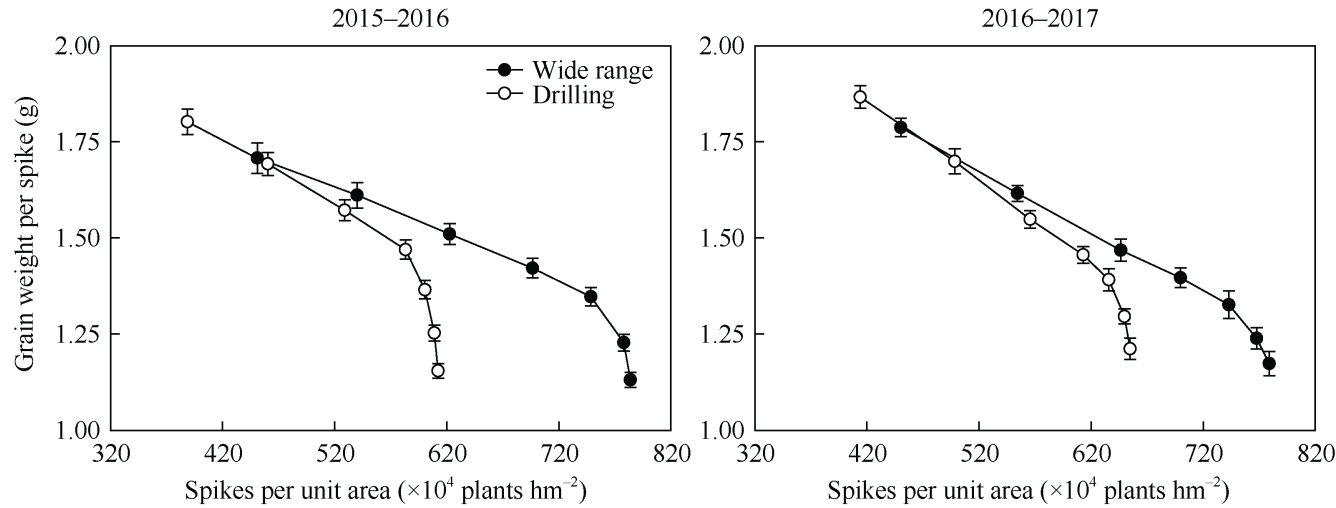

图 3 播种方式与种植密度互作对大穗型小麦品种穗数和单穗粒重的影响

Fig. 3 Effect of interaction of sowing pattern and planting density on the spikes per unit area and the grain weight per spike of large spike wheat cultivar

误差线表示 3 次重复的标准误。

The error bars represent standard error of three replicates.

2.5 宽幅播种与种植密度互作对冬小麦氮素吸 收效率和氮素内在利用效率的影响

如图 4 所示, 与相邻低密度的氮素吸收效率相 比，常规条播下密度由 $130 \times 10^{4}$ 株 $\mathrm{hm}^{-2}$ 增至 $200 \times$ $10^{4} 、 270 \times 10^{4} 、 340 \times 10^{4}$ 株 $\mathrm{hm}^{-2}$, 氮素吸收效率由 $52.28 \%$ 分别增至 $60.13 \% 、 65.84 \%$ 和 $69.91 \%$ ，两生长 季氮素吸收效率绝对值每增加 $1 \%$, 氮素内在利用效率 相应下降 $0.25 、 0.19$ 和 $0.17 \mathrm{~kg} \mathrm{~kg}^{-1}$; 密度进一步由 $340 \times 10^{4}$ 株 $\mathrm{hm}^{-2}$ 增至 $410 \times 10^{4} 、 480 \times 10^{4} 、 550 \times 10^{4}$ 株 $\mathrm{hm}^{-2}$, 氮素吸收效率由 $69.91 \%$ 依次降至 $68.42 \%$ 、 $65.83 \%$ 与 $63.39 \%$, 氮素内在利用效率相应下降 0.26 、 0.56 和 $0.81 \mathrm{~kg} \mathrm{~kg}^{-1}$ 。宽幅播种下, 密度由 $130 \times 10^{4}$ 株 $\mathrm{hm}^{-2}$ 增至 $410 \times 10^{4}$ 株 $\mathrm{hm}^{-2}$, 两生长季氮素吸收效率由 $59.58 \%$ 增至 $70.30 \% 、 77.75 \% 、 83.62 \%$ 与 $85.99 \%$ ，氮 素吸收效率绝对值每增加 $1 \%$ ，氮素内在利用效率分别 下降 $0.18 、 0.12 、 0.11$ 和 $0.09 \mathrm{~kg} \mathrm{~kg}^{-1}$ ，种植密度进一步 由 $410 \times 10^{4}$ 株 $\mathrm{hm}^{-2}$ 增至 $550 \times 10^{4}$ 株 $\mathrm{hm}^{-2}$, 氮素吸收效 率由 $85.99 \%$ 降至 $82.69 \%$ 和 $78.77 \%$ ，氮素内在利用效率 分别下降 $0.06 \mathrm{~kg} \mathrm{~kg}^{-1}$ 和 $0.13 \mathrm{~kg} \mathrm{~kg}^{-1}$ 。

\section{3 讨论}

\section{1 宽幅播种与增密配套进一步提高冬小麦产量} 小麦的产量受控于群体数量和个体生产力两 个方面。一般认为, 随单位面积穗数增加单穗粒重

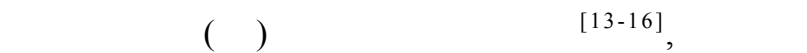
增加单位面积穗数的同时尽可能减轻单穗粒重的 降低幅度才能获得更高产量 ${ }^{[16-17]}$ 。前人研究表明, 在一定范围内增加密度可以提高小麦产量, 继续 增加密度则会显著减产 ${ }^{[18-19]}$, 且通常获得最高产 量的密度多在 $225 \times 10^{4}$ 株 $\mathrm{hm}^{-2}$ 与 $375 \times 10^{4}$ 株 $\mathrm{hm}^{-2}$ 之间 ${ }^{[21-23]}$, 相应穗数多集中在 $515 \times 10^{4}$ 与 $660 \times 10^{4}$ 穗 $\mathrm{hm}^{-2}$ 范围内 ${ }^{[13,20]}$, 这与本试验中常规条播下 $340 \times 10^{4}$ 株 $\mathrm{hm}^{-2}$ 密度 $\left(596.15 \times 10^{4}\right.$ 穗 $\left.\mathrm{hm}^{-2}\right)$ 获得最高 产量的结果基本一致。然而，宽幅播种下获得最高 产量的密度为 $410 \times 10^{4}$ 株 $\mathrm{hm}^{-2}$, 相应穗数为 $745.81 \times 10^{4}$ 穗 $\mathrm{hm}^{-2}$, 显著高于前人和本试验中常规 条播的最适密度和容穗量。分析其原因在于改常规 条播为宽幅播种, 虽然随密度增加穗粒数与千粒重 
2015-2016

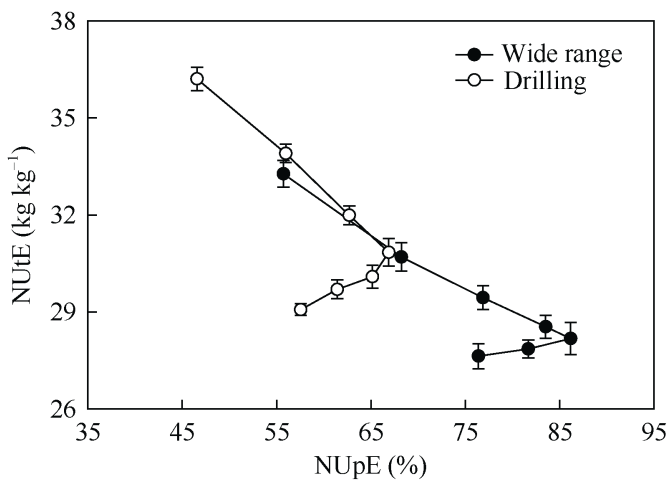

2016-2017

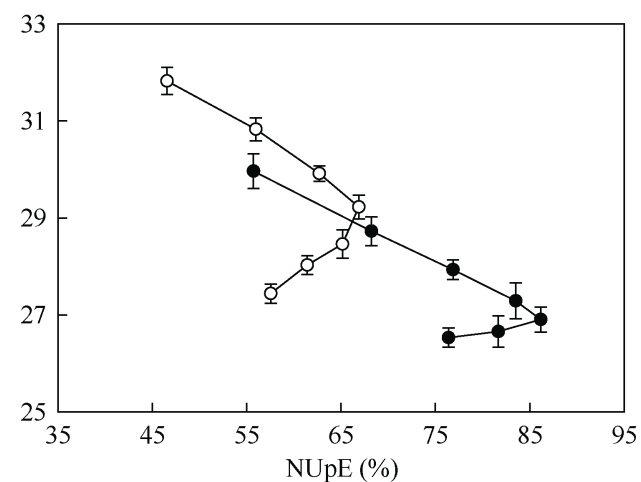

图 4 播种方式与种植密度互作对大穗型小麦品种氮素吸收效率和氮素内在利用效率的影响

Fig. 4 Effect of interaction of sowing pattern and planting density on the nitrogen uptake efficiency (NUpE) and nitrogen utilization efficiency (NUtE) of large spike wheat cultivar

误差线表示 3 次重复的标准误。

The error bars represent standard error of three replicates.

均呈下降趋势, 但其降幅显著低于常规条播的同密度 处理, 相同单穗粒重条件下宽幅播种处理的容穗量显 著高于常规条播, 而且当密度为 $410 \times 10^{4}$ 株 $\mathrm{hm}^{-2}$ 、穗 数达 $745.81 \times 10^{4}$ 穗 $\mathrm{hm}^{-2}$ 后, 宽幅播种的单穗粒重才 快速下降，而常规条播下密度为 $340 \times 10^{4}$ 株 $\mathrm{hm}^{-2}$ 、 穗数达到 $596.15 \times 10^{4}$ 穗 $\mathrm{hm}^{-2}$ 后单穗粒重就出现了 急剧下降。表明宽幅播种与适宜密度相匹配, 可在 较高穗数时保持较高的单穗粒重, 从而奠定了进一 步增产的群体数量与个体生产力基础。

3.2 宽幅播种与增密配套进一步提高冬小麦氮 素利用率

前人研究表明, 增加密度可以提高小麦氮素利 用率, 但获得最高氮素利用率的密度多在 $225 \times 10^{4}$ 株 $\mathrm{hm}^{-2}$ 与 $375 \times 10^{4}$ 株 $\mathrm{hm}^{-2}$ 之间 ${ }^{[15,22]}$, 这与本试验 中常规条播下的情况类似。密度继续增加则会导致 氮素利用率显著降低 ${ }^{[15,22]}$ 。与常规条播不同, 宽幅播 种下获得最优氮素利用率的密度为 $410 \times 10^{4}$ 株 $\mathrm{hm}^{-2}$, 显著高于前人和本试验常规条播的最适密度, 且其 增效幅度亦显著高于常规条播, 从而表明宽幅播种 与增密配套具有进一步提高氮素利用率的潜力。

氮素利用率的高低取决于氮素吸收效率和氮素 内在利用效率的协调程度 ${ }^{[11,24-25]}$ 。在同样的供氮水 平下，氮素吸收效率的提高往往会导致氮素内在利 用效率不同程度的降低，两者的协调利于氮素利用 率的稳定 ${ }^{[26]}$ 或进一步提高 ${ }^{[2,27]}$ 。在本研究中, 宽幅播 种与适宜密度合理匹配, 在同样密度下随氮素吸收 效率提高, 氮素内在利用效率的降低幅度不仅低于 常规条播, 而且在密度为 $410 \times 10^{4}$ 株 $\mathrm{hm}^{-2}$ 时亦显著 低于传统条播下密度为 $340 \times 10^{4}$ 株 $\mathrm{hm}^{-2}$ 的处理, 有
效缓解了氮素吸收效率提高与氮素内在利用效率降 低的矛盾，有利于促进氮素利用率的进一步提高。 同时我们注意到，在获得最优氮素吸收效率之后， 进一步提高密度时氮素吸收效率和氮素内在利用效 率同步下降，这表明宽幅播种与增密配套提高氮素 利用率也是有限度的。

\section{4 结论}

与传统条播相比，宽幅播种配合增密既促进了 单位面积穗数和氮素吸收效率的增加, 又缓解了单 穗粒重与氮素内在利用效率的降低幅度, 进而获得 更高的籽粒产量和氮素利用率。在本试验条件下, 对大穗型小麦品种泰农 18 来说, 宽幅播种(苗带宽 $8 \sim 10 \mathrm{~cm})$ 与 $410 \times 10^{4}$ 株 $\mathrm{hm}^{-2}$ 密度相匹配是获得更高 产高效的最优组合。

\section{References}

[1] Tokatlidis I S. Addressing the yield by density interaction is a prerequisite to bridge the yield gap of rain-fed wheat. Ann Appl Biol, 2014, 165: 27-42.

[2] Dai X L, Zhou X H, Jia D Y, Xiao L L, Kong H B, He M R. Managing the seeding rate to improve nitrogen-use efficiency of winter wheat. Field Crops Res, 2013, 154: 100-109.

[3] Dai X L, Xiao L L, Jia D Y, Kong H B, Wang Y C, Li C X, Zhang Y, He M R. Increased plant density of winter wheat can enhance nitrogen-uptake from deep soil. Plant Soil, 2014, 384: 141-152.

[4] 冯伟, 李世荣, 王永华, 康国章, 段剑钊, 郭天财. 宽幅播种 下带间距对冬小麦衰老进程及产量的影响. 生态学报, 2015, 35: 2686-2694.

Feng W, Li S Y, Wang Y H, Kang G Z, Duan J Z, Guo T C. Effects of spacing intervals on the ageing process and grain yield in winter wheat under wide bed planting methods. Acta Ecol Sin, 2015, 35: 2686-2694 (in Chinese with English abstract). 
[5] 李世芗, 冯伟, 王永华, 王晨阳, 郭天财. 宽幅播种带间距对 冬小麦冠层特征及产量的影响. 植物生态学报, 2013, 37: $758-767$.

Li S Y, Feng W, Wang Y H, Wang C Y, Guo T C. Effects of spacing interval of wide bed planting on canopy characteristics and yield in winter wheat. Chin J Plant Ecol, 2013, 37: 758-767 (in Chinese with English abstract).

[6] 初金鹏, 朱文美, 尹立俊, 石玉华, 邓淑珍, 张良, 贺明荣, 代 兴龙. 宽幅播种对冬小麦“泰农 18 '产量和氮素利用率的影响. 应用生态学报, 2018, 29: 2517-2524.

Chu J P, Zhu W M, Yin L J, Shi Y H, Deng S Z, Zhang L, He M R, Dai X L. Effects of wide-range planting on the yield and nitrogen use efficiency of winter wheat cultivar Tainong 18. Chin J Appl Ecol, 2018, 29: 2517-2524 (in Chinese with English abstract).

[7] 石玉华, 初金鹏, 尹立俊, 贺明荣, 邓淑珍, 张良, 孙晓乐, 田 奇卓, 代兴龙. 宽幅播种提高不同播期小麦产量与氮素利用 率. 农业工程学报, 2018, 34(17): 127-133.

Shi Y H, Chu J P, Yin L J, He M R, Deng S Z, Zhang L, Sun X Y, Tian Q Z, Dai X L. Wide-range sowing improving yield and nitrogen use efficiency of wheat sown at different dates. Trans CSAE, 2018, 34(17): 127-133 (in Chinese with English abstract).

[8] 杨文平, 郭天财, 刘胜波, 王晨阳, 王永华, 马冬云. 行距配 置对“兰考矮早八’小麦后期群体冠层结构及其微环境的影响. 植物生态学报, 2008, 32: 485-490.

Yang W P, Guo T C, Liu S B, Wang C Y, Wang Y H, Ma D Y. Effects of row spacing in winter wheat on canopy structure and microclimate in later growth stage. Chin J Plant Ecol, 2008, 32: 485-490 (in Chinese with English abstract).

[9] 郭天财, 刘胜波, 冯伟, 杨文平, 王永华, 韩巧霞. 不同种植 行距的大穗型小麦品种“兰考矮早八’中几种与旗叶衰老有关 的生理指标变化. 植物生理学通讯, 2008, 44: 33-36.

Guo T C, Liu S B, Feng W, Yang W P, Wang Y H, Han Q X. Changes in several physiological indices related to senescence in flag leaves of heave-ear wheat 'Lankao'aizao 8' with different row spacing. Plant Physiol Commum, 2008, 44: 33-36 (in Chinese with English abstract).

[10] 郭天财, 盛坤, 冯伟, 徐丽娜, 王晨阳. 种植密度对两种穗型 小麦品种分藮期茎藮生理特性的影响. 西北植物学报, 2009, 29: 350-355.

Guo T C, Sheng K, Feng W, Xu L N, Wang C Y. Effects of plant density on physiological characteristics of different stems during tillering stage in two spike-types winter wheat cultivars. Acta Bot Boreal-Occident Sin, 2009, 29: 350-355 (in Chinese with English abstract).

[11] Moll R H, Kamprath E J, Jackson W A. Analysis and interpretation of factors which contribute to efficiency to nitrogen utilization. Agron J, 1982, 75: 562-564.

[12] 申建波, 毛达如. 植物营养学研究方法(第 3 版). 北京: 中国 农业大学出版社, 2011. pp 52-53.

Shen J B, Mao D R. Research Methods of Plant Nutrition, 3rd edn. Beijing: China Agricultural University Press, 2011. pp 52-53 (in Chinese).

[13] Ma S C, Wang T C, Guan X K, Zhang X. Effect of sowing time and seeding rate on yield components and water use efficiency of winter wheat by regulating the growth redundancy and physiological traits of root and shoot. Field Crops Res, 2018, 221: 166-174.

[14] Melash A A, Mengistu D K, Aberra D A, Tsegay A. The influence of seeding rate and micronutrients foliar application on grain yield and quality traits and micronutrients of durum wheat. $J$ Cereal Sci, 2019, 85: 221-227.

[15] Tao Z Q, Ma S K, Chang X H, Wang D M, Wang Y J, Yang Y S, Zhao G C, Yang J C. Effects of tridimensional uniform sowing on water consumption, nitrogen use, and yield in winter wheat. Crop $J, 2019.7: 480-493$.

[16] Zhu Y G, Chu J P, Dai X L, He M R. Delayed sowing increases grain number by enhancing spike competition capacity for assimilates in winter wheat. Eur J Agron, 2019, 104: 49-62.

[17] Chen C, Neill K, Wichman D, Westcott M. Hard red spring wheat response to row spacing, seeding rate, and nitrogen. Agron J, 2008, 100: 1296-1302.

[18] Carr P M, Horsley R D, Poland W W. Tillage and seeding rate effects on wheat cultivars: I. Grain production. Crop Sci, 2003, 43: 202-209.

[19] Lloveras J, Manent J, Viudas J, López A, Santiveri P. Seeding rate influence on yield and yield components of irrigated winter wheat in a Mediterranean climate. Agron J, 2004, 96: 1258-1265.

[20] 张永丽, 肖凯, 李雁鸣. 种植密度对杂种小麦 C6-38/Py85-1 旗 叶光合特性和产量的调控效应及其生理机制. 作物学报, 2005, 31: 498-505.

Zhang Y L, Xiao K, Li Y M. Effects and physiological mechanism of planting densities on photosynthesis characteristics of flag leaf and grain yield in wheat hybrid C6-38/Py85-1. Acta Agron Sin, 2005, 31: 498-505 (in Chinese with English abstract).

[21] 陈雨海, 余松烈, 于振文. 小麦生长后期群体光截获量及其分 布与产量的关系. 作物学报, 2003, 29: 730-734.

Chen Y H, Yu S L, Yu Z W. Relationship between amount or distribution of PAR interception and grain output of wheat communities. Acta Agron Sin, 2003, 29: 730-734 (in Chinese with English abstract).

[22] Yang D Q, Cai T, Luo Y L, Wang Z L. Optimizing plant density and nitrogen application to manipulate tiller growth and increase grain yield and nitrogen-use efficiency in winter wheat. Peer J: Life Environ, 2019, 7: e6468.

[23] 曹倩, 贺明荣, 代兴龙, 门洪文, 王成雨. 密度、氮肥互作对小 麦产量及氮素利用效率的影响. 植物营养学与肥料学报, 2011, 17: 815-822.

Cao Q, He M R, Dai X L, Men H W, Wang C Y. Effects of interaction between density and nitrogen on grain yield and nitrogen use efficiency of winter wheat. Plant Nutr Fert Sci, 2011, 17: 815-822 (in Chinese with English abstract).

[24] Foulkes M J, Hawkesford M J, Barraclough P B, Holdsworth M J, Kerr S, Kightley S, Shewry P R. Identifying traits to improve the nitrogen economy of wheat: Recent advances and future prospects. Field Crops Res, 2009, 114: 329-342.

[25] Salvagiotti F, Castellarin J M, Miralles D J, Pedrol H M. Sulfur fertilization improves nitrogen use efficiency in wheat by increasing nitrogen uptake. Field Crops Res, 2009, 113: 170-177.

[26] Dai X L, Wang Y C, Dong X C, Qian T F, Yin L J, Dong S X, Chu J P, He M R. Delayed sowing can increase lodging resistance while maintaining grain yield and nitrogen use efficiency in winter wheat. Crop J, 2017, 5: 541-552.

[27] Sadras V O, Lawson C. Nitrogen and water-use efficiency of Australian wheat varieties released between 1958 and 2007. Eur J Agron, 2013, 46: 34-41. 